\title{
La megabiblioteca José Vasconcelos y la situación de las bibliotecas públicas de la Ciudad de México
}

\section{María de los Ángeles Ciprés Oliva Alva Flores Romero}

Biblioteca del Instituto de Investigaciones Filológicas de la UNAM (México)

\section{Resumen}

El objetivo de este trabajo es demostrar que, dadas las condiciones actuales de las bibliotecas públicas en la ciudad de México, es ilógico pensar en la construcción de una gran biblioteca, pues esta absorberá el presupuesto indispensable para el mejoramiento de las ya existentes. Se utilizó el análisis de la información contenida en las fuentes bibliohemerográficas relacionadas con el tema, la observación directa para compilar datos y conocer las condiciones actuales de las bibliotecas, y los cuestionarios respondidos por los encargados de las bibliotecas públicas delegacionales de la Ciudad de México. Muchas de las bibliotecas necesitan mejorar sus instalaciones, ya que no tienen luz suficiente, no cuentan con un buen servicio de sanitarios y en general carecen de los elementos necesarios para ofrecer un espacio agradable. En las bibliotecas públicas de la Ciudad de México existen necesidades básicas e importantes que aún no son atendidas por falta de recursos económicos, por lo que resulta ilógico invertir en la construcción de la nueva megabiblioteca José Vasconcelos. El Consejo Nacional para la Cultura y las Artes (Conaculta) debería establecer prioridades con base en las necesidades reales de la Red Nacional de Bibliotecas Públicas.

Palabras clave: Bibliotecas públicas. Aspectos políticos. Evaluación. Ciudad de México.

\section{Abstract}

We attempt to demonstrate, according to the present-day conditions of public libraries in Mexico City, how illogical is thinking about the creation of a large library, which will use up most of the financial budget required for the improvement of current libraries. Three methodological approaches where used: the analysis of the information included in the bibliographical sources as well as in the periodical publishings (newspapers and magazines), the direct observation to compile data as well as being acquainted with the current situation of the libraries, and the application 
of tests to the staff in the community libraries that belong to the different districts of Mexico City. As a result, it was stated that many libraries require better installations, as electricity service is inadequate, and libraries lack adequate plumbing (rest rooms). In general, libraries need the essential elements to create a pleasant environment. So, it was concluded that there are essential and important necessities in community libraries, which have not been taken into account because of the lack of financial resources. In consequence it would be illogical to invest in the creation of the new large library José Vasconcelos. CONACULTA should establish priorities based in the real needs of the Public Libraries National Network (Red Nacional de Bibliotecas Publicas).

Keywords: Public libraries. Political aspects. Evaluation. Mexico City.

\section{Introducción}

La presente investigación surgió a partir de una serie de trabajos realizados acerca de la Red Nacional de Bibliotecas Públicas de la ciudad de México, durante los cuales nos percatamos de las carencias y problemas que presentan casi todas ellas, problemas que afectan directamente al servicio y a la afluencia de usuarios, y que lamentablemente se ven reflejados en una escasa asistencia a las bibliotecas públicas.

Cuando se dio a conocer el Plan Nacional de Desarrollo 2001-2006 fue muy alentador saber que, a raíz de un diagnóstico realizado a la Red Nacional de Bibliotecas Públicas, se plantearon planes y proyectos encaminados a subsanar sus deficiencias y de esta manera mejorar el servicio que proporcionan.

Sin embargo, cuando se dio a conocer el proyecto de la construcción de la megabiblioteca José Vasconcelos nos dimos cuenta de que, lejos de dar seguimiento a los planes principales de mejorar las condiciones de funcionamiento de las bibliotecas, se estaba planteando la construcción de un nuevo edificio para la Biblioteca de México José Vasconcelos, que funge actualmente de biblioteca central de la Red Nacional de Bibliotecas Públicas. Esto fue en muchos aspectos desalentador, pues conocemos de cerca todos los problemas que han de afrontar estas instituciones y creemos que la construcción de este edificio no va a resolverlos, como tampoco solucionará la cuestión de la falta de lectura entre la población. Asimismo, pensamos que la solución debería darse mediante una atención efectiva a las bibliotecas ya existentes, dotándolas de todos los recursos materiales y humanos para lograr fomentar un hábito tan importante como es la lectura en la comunidad a la que sirven.

Por todo lo expuesto, iniciamos esta investigación con el objetivo de demostrar que, dadas las condiciones actuales de las bibliotecas públicas en la Ciudad de México, es ilógico pensar en la construcción de una gran biblioteca, pues esta absorberá el presupuesto indispensable para el mejoramiento de las ya existentes.

Scire. $13: 1$ (en.-jun. 2007) 51-66. ISSN 1135-3716. 
Para cumplir con el objetivo planteado, el presente trabajo se estructuró en cuatro apartados: la primera parte explica la metodología utilizada para llevar a cabo el estudio; la segunda da a conocer, como marco de referencia, el proyecto de la nueva megabiblioteca, describiendo sus antecedentes y objetivos a fin de tener un margen de descripción; en la tercera se expone la situación actual de las bibliotecas públicas en la Ciudad de México, mostrando las condiciones en las que se encuentran y el punto de vista de los encargados de estas bibliotecas con respecto al nuevo proyecto; la cuarta parte analiza los resultados obtenidos en este estudio. Por último se presentan las conclusiones, las recomendaciones, la bibliografía, y el anexo, que consiste en el cuestionario aplicado a los encargados de las bibliotecas públicas de las redes delegacionales de la Ciudad de México.

\section{Metodología}

Para cumplir con el objetivo planteado al inicio de este estudio se consideró apropiado aplicar la metodología que exponemos a continuación.

El primer método utilizado fue el análisis de la información contenida en las fuentes bibliohemerográficas relacionadas, por un lado, con la situación actual de las bibliotecas públicas en la Ciudad de México, y, por otro, con el proyecto de la megabiblioteca José Vasconcelos, para conocer el modo en que surgió este proyecto y los propósitos de su creación.

Por tratarse de una investigación descriptiva, para obtener la información requerida se utilizaron técnicas basadas en cuestionarios, entrevistas y observación, las cuales fueron aplicadas a los coordinadores de las redes delegaciones de bibliotecas públicas de la Ciudad de México para conocer su punto de vista acerca del proyecto de la nueva megabiblioteca, y de esta manera enterarnos de las carencias que existen en la Red de Bibliotecas Públicas de la ciudad.

Es importante mencionar que, dado que el cuestionario tiene una cantidad pequeña de preguntas, el recuento y la decodificación de las respuestas obtenidas se realizó de forma manual, y los medios automatizados solo se utilizaron para graficar y presentar los resultados.

Debido a la dificultad que representaba estudiar a toda la Red de Bibliotecas Públicas de la Ciudad de México, se prefirió visitar las bibliotecas centrales de cada una de las 16 delegaciones que la conforman, ya que, de acuerdo con la función que desempeñan, fueron idóneas para obtener la información necesaria para la realización de la investigación. Estas 16 delegaciones son Álvaro Obregón, Azcapotzalco, Benito Juárez, Coyoacán, Cuajimalpa de Morelos, Cuauhtémoc, Gustavo A. Madero, Iztacalco, Iztapalapa, Magdalena Contreras, Milpa Alta, Miguel Hidalgo, Tláhuac, Tlalpan, Venustiano Carranza y Xochimilco.

Scire. $13: 1$ (en.-jun. 2007) 51-66. ISSN 1135-3716. 
La técnica de campo básica que se utilizó fue la de la encuesta, y para cumplir con su propósito en esta investigación el cuestionario aplicado tuvo las siguientes características: $a$ ) fue aplicado personalmente bajo la modalidad de entrevista con el objeto de que la observación directa complementara la información verbal proporcionada por los encuestados; $b$ ) tuvo un formato preestablecido; $c$ ) debido a que se requerían algunas opiniones y sugerencias de los encuestados, y tomando en cuenta que este tipo de información es difícil de codificar en opciones, en los cuestionarios se incluyeron preguntas abiertas o no estructuradas, que son aquellas que "permiten a los encuestados contestar libremente sin tener que seleccionar una de varias respuestas proporcionadas" (Busha, 1990, p. 72); $d$ ) se incluyeron preguntas sobre opinión y actitud para conocer el punto de vista de los encuestados sobre el proyecto de la megabiblioteca José Vasconcelos.

En cuanto a la aplicación de los cuestionarios, las bibliotecas fueron elegidas tomando como fuente básica el Directorio de Bibliotecas del Distrito Federal, editado por el Consejo Nacional para la Cultura y las Artes (en adelante Conaculta); de él se seleccionaron las bibliotecas centrales delegacionales por ser las coordinadoras de todas las bibliotecas públicas que conforman la red delegacional, por lo que toda la información acerca de las condiciones en las que se encuentran las bibliotecas de la delegación es manejada por ellas. Además, debido a su rango de coordinación reciben mayor información acerca de los planes y proyectos de la Dirección General de Bibliotecas (en adelante DGB) y de la Secretaría de Educación Pública (SEP) con el fin de que estas la difundan a todas las bibliotecas que coordinan.

Con respecto al horario y a los días de la semana en que se aplicaron los cuestionarios, podemos decir que este trabajo se llevó a cabo por la mañana, de lunes a viernes, tomando en cuenta que había mayor posibilidad de localizar a los encargados. Cabe mencionar que esta actividad se realizó durante mes y medio.

\section{La megabiblioteca José Vasconcelos}

El edificio que ocupa actualmente la Biblioteca de México José Vasconcelos, conocido como la Ciudadela, se empezó a construir en 1792 y ha albergado a distintas instituciones, desde la Real Fábrica de Tabaco hasta un almacén de medicinas de Sanidad Militar, y fue declarado monumento histórico en 1931.

La Biblioteca de México José Vasconcelos fue fundada el 27 de noviembre de 1946 por José Vasconcelos, y desde su creación ha proporcionado, además del servicio escolar, el préstamo de fondos conventuales del siglo XIX, material donado por grandes bibliófilos y coleccionistas particulares, como Joaquín García Icazbalceta, Antonio Caso y Jesús Reyes Heroles, entre otros.

En el año de 1987 se le otorgó el grado de Centro Bibliotecario Nacional, Biblioteca Pública de México, de la Red Nacional de Bibliotecas Públicas, con los 
objetivos de ser el núcleo coordinador y dotar de libros para el servicio de préstamo interbibliotecario a toda la Red, lo que hasta ahora no ha cumplido por falta de recursos económicos.

En 1988 la biblioteca fue remodelada completamente y distribuida en diferentes patios para que funcionara como centro de cultura. Desde entonces proporciona servicios tales como talleres de lectura y de teatro, cine club, servicio para invidentes y lectura al aire libre. Actualmente la novedad tecnológica más representativa del lugar es su catálogo en línea.

El proyecto de la megabiblioteca José Vasconcelos, que consiste en la construcción de un nuevo edificio para la Biblioteca Pública de México, surge a partir del Plan Nacional de Cultura 2001-2006, que contempla, entre otros objetivos, el mejoramiento de toda la infraestructura bibliotecaria del país. Este proyecto es planteado en el programa nacional Hacia un país de lectores, en el cual se habla formalmente de "la creación de un nuevo paradigma de biblioteca pública" ("Se instaló el Comité Consultivo...”, 2003, p. 2).

Este último plan fue dado a conocer en diciembre del 2002 por el secretario de la SEP, Reyes Tamez Guerra, y la presidenta del Conaculta, Sari Bermúdez, como un proyecto de modernización de la Biblioteca Pública Central de la Red Nacional de Bibliotecas Públicas, conocida como Biblioteca de México José Vasconcelos.

Las entidades responsables de la ejecución y coordinación operativa del proyecto son la SEP y el Conaculta, las que además están apoyadas por cuatro órganos: el comité consultivo, un patronato, un fideicomiso público y un comité técnico. En el primero de ellos se encuentran representantes de las más importantes instituciones especializadas en el campo de la bibliotecología, la arquitectura, el libro y la lectura, y la tecnología; el patronato se encarga de la recaudación de fondos y donativos para la construcción y el equipamiento del edificio; el fideicomiso se encargará de la aplicación ágil, transparente y eficiente de los recursos económicos, y, finalmente, el comité técnico será el responsable de la canalización de recursos y la supervisión del proyecto.

Para seleccionar el proyecto arquitectónico de la nueva sede de la Biblioteca José Vasconcelos, se convocó un Concurso Internacional de Arquitectura en el que participaron 592 propuestas y donde resultaron ganadores el mexicano Alberto Kalach y su equipo.

La principal particularidad de esta propuesta arquitectónica consiste en el aprovechamiento de la infraestructura para desarrollar un área verde, un jardín botánico que abarcará terrenos de la biblioteca y espacios colindantes. El edificio estará compuesto

Scire. $13: 1$ (en.-jun. 2007) 51-66. ISSN 1135-3716. 
por tres naves, cada una de ellas de 90 metros de largo, que en conjunto sumarán cerca de 300 metros de largo, con 36 metros de ancho y casi 25 metros de altura. Los libros estarán situados en una serie de libreros colgantes, ubicados en la parte central de la estructura, formando una especie de espina dorsal, que permitirá recorrer todo el edificio, y tendrá cuatro niveles, dos de ellos subterráneos. ("Se presentó el proyecto de la nueva Biblioteca...", 2003, p. 6)

Las nuevas instalaciones tendrán unos objetivos generales:

Mejorar la calidad de los servicios que ofrece el sistema bibliotecario nacional.

Brindar a toda la población acceso gratuito a los distintos medios y fuentes de información incluyendo los desarrollados por las nuevas tecnologías de la información y la comunicación. (ibídem, p. 2)

Y unos objetivos específicos:

Ser el centro de cómputo o de servicios electrónicos de la Red, con la infraestructura de telecomunicaciones e informática que habrá a nivel nacional en el mediano y largo plazos. ("La editorial alemana Taschen...", 2004)

Ser el centro de servicios a distancia para las bibliotecas estatales, regionales y municipales de toda la Red". ("Editorial: la nueva sede...", 2003)

El costo de la construcción de la megabiblioteca había sido estimado en 60 millones de dólares en 2002, pero la última cifra calculada se encuentra entre los 80 y los 100 millones de dólares. Se espera lograr que el $50 \%$ de este dinero provenga de recursos fiscales federales, y el otro $50 \%$ de donativos, tanto públicos como privados. Del total, 65 millones se invertirán en el nuevo edificio y el resto en la adquisición de las colecciones.

Cabe mencionar que en mayo del 2002 se dio a conocer el convenio de colaboración entre el Gobierno mexicano, la Fundación Bill y Melinda Gates de los Estados Unidos y la empresa Microsoft de México, que consiste en un donativo de 30 y 10 millones de dólares, respectivamente, para el equipamiento de bibliotecas públicas en todo el país, principalmente para aquellas que atiendan a comunidades marginadas, esto con el objetivo fundamental de ofrecer servicios digitales gratuitos a personas de escasos recursos. De acuerdo con Jorge von Ziegler, director de la Red Nacional de Bibliotecas Públicas, este donativo no se va a aplicar en ningún porcentaje a la construcción de la megabiblioteca, "sino que servirá para la infraestructura tecnológica y de cómputo que requieren las bibliotecas pertenecientes a la red nacional” (“Anuncian partida de \$250 millones...", 2003).

Las nuevas instalaciones de la Biblioteca México José Vasconcelos estarán localizadas en la Ciudad de México, en los terrenos ubicados en la antigua área de la estación de ferrocarriles Buenavista, en la zona norte de la capital del país. Además, a esta superficie se agregarán terrenos pertenecientes a Ferrocarriles Nacionales de México, los cuales Banobras enajenará a favor de la SEP y el Conaculta para la construcción de la megabiblioteca. La superficie total que ocupará el in- 
mueble será de un rango de 32000 a 35000 metros cuadrados y, según los cálculos, podrá albergar más de 2 millones de volúmenes. Las salas de las que constará este inmueble son una sala de lectura, un laboratorio de idiomas, un museo virtual, una hemeroteca, un auditorio, un centro comunitario digital e-México y un restaurante.

Los servicios que prestará la megabiblioteca en estas nuevas instalaciones serán, además de los que hasta el momento tiene encomendados, las funciones que se deriven de convertirse en la biblioteca central de la Red Nacional de Bibliotecas Públicas y de "marcar las pautas metodológicas del sistema bibliotecario nacional", ("Requiere especialistas...", 2002), esto en cuanto a la calidad y eficiencia en el servicio a los usuarios, así como en la tarea de establecer normas para la selección de materiales y la organización de los fondos.

En cuanto al acervo que conformará a la megabiblioteca, las colecciones se formarán siguiendo los criterios de incluir los materiales más actualizados y de todo tipo de información de cada disciplina (según el sistema de clasificación Dewey), así como los fondos editoriales de más prestigio en el mundo, y particularmente los de lengua española. Para lograr todo lo anterior, y para enriquecer las colecciones, el Conaculta dio inicio a una campaña de donativos de libros con las más importantes editoriales de México y el extranjero, donativos que se sumarán a los materiales adquiridos por el propio Conaculta. La colección inicial será de aproximadamente 500000 ejemplares, de los cuales se calcula que al menos el $20 \%$ procederán de donaciones y el resto serán adquiridos.

Es muy importante mencionar que este proyecto contempla tanto el inmueble de la Ciudadela como el nuevo edificio; "no se trata de una nueva institución, sino de un nuevo edificio para una biblioteca que ya existe" ("Se presentó el proyecto de la nueva Biblioteca...", 2003, p. 6). Por lo tanto, se está realizando una revisión de qué acervo y qué funciones se quedan en la Ciudadela y cuáles se llevarán al edificio de Buenavista. El planteamiento inicial es que en la primera se concentren los materiales y servicios enfocados a los investigadores y las áreas administrativas de la DGB del Conaculta, en tanto que el nuevo edificio se enfocaría al público en general.

\section{La situación actual de las bibliotecas públicas de la Ciudad de México}

Antes de dar a conocer la situación de las bibliotecas públicas de la Ciudad de México, consideramos conveniente hablar de la estructura y el funcionamiento de la Red Nacional de Bibliotecas Públicas. 


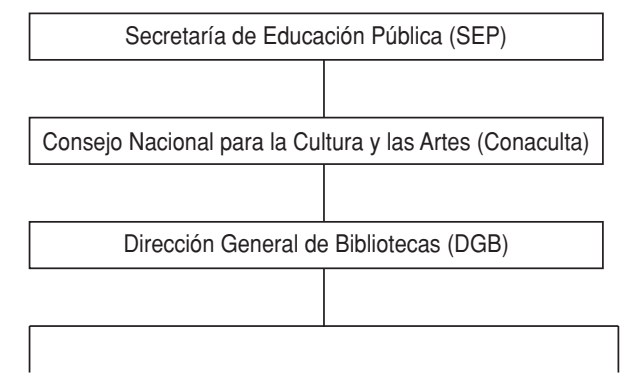

En cada estado de la República:

En la Ciudad de México:
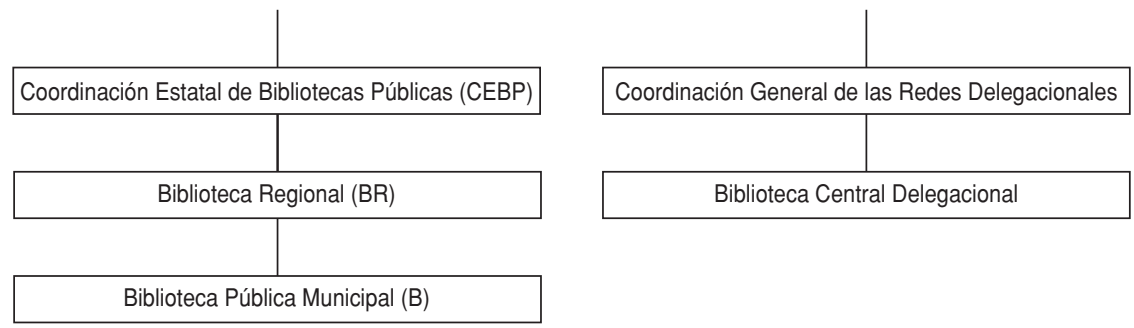

La SEP es el órgano máximo de coordinación de la Red, y realiza esta labor a través del Conaculta, que a su vez delega esta función en la DGB ("La Red Nacional de Bibliotecas Públicas", 1991, p. 9).

A partir de estas instancias se implementaron una serie de coordinaciones a lo largo de todo el país, las cuales se encuentran distribuidas de la siguiente forma: a) en cada uno de los 31 estados de la República existe una Coordinación Estatal de Bibliotecas Públicas, representada por una biblioteca pública central estatal, la cual coordina la Red de Bibliotecas Públicas de todo el estado; $b$ ) en un nivel inferior de coordinación se encuentran las bibliotecas regionales, que son las bibliotecas públicas de las ciudades más importantes de cada estado y cuya función es "servir de enlace entre la biblioteca central estatal y las municipales" ("El programa Nacional de Bibliotecas Públicas 1983-1988...”, 1988, p. 26); c) las bibliotecas públicas municipales constituyen el último nivel de coordinación de la Red en cada estado, y tienen a su cargo las bibliotecas de menor rango pertenecientes a pueblos y barrios.

Podemos destacar que en cierta forma la Ciudad de México constituye un caso aparte, ya que la coordinación de sus bibliotecas públicas está a cargo de organismos diferentes a los que coordinan las bibliotecas en el interior de la República; sin embargo, dichos organismos constituyen niveles de coordinación equivalentes. De esta forma tenemos que la coordinación general de las 16 redes delegacionales está a cargo de la Dirección de Operación de Bibliotecas Públicas. 
Por su parte, cada una de las 16 delegaciones políticas cuenta con una biblioteca central delegacional, la cual está subordinada a la Jefatura de Unidad de Bibliotecas Públicas de Zona (Poniente y Oriente), cuya función es coordinar a todas las bibliotecas públicas que conforman la red delegacional. Esta Jefatura depende de la Subdirección de Bibliotecas Públicas del Distrito Federal, que a su vez está supeditada a la Dirección de Operación de Bibliotecas Públicas.

\section{Resultados}

Después de conocer algunos de los pormenores del proyecto de la construcción de la megabiblioteca José Vasconcelos y de la Red Nacional de Bibliotecas Públicas, vamos hablar de la situación de las bibliotecas públicas de la Ciudad de México (según la literatura consultada y la observación directa), la cual, lamentablemente, no es muy buena por varias razones.

En primer lugar, el nivel de afluencia a la mayor parte de ellas es muy bajo, y de los pocos visitantes que reciben el mayor porcentaje está representado por estudiantes de primaria y secundaria, los usuarios de otro tipo son casi inexistentes en sus instalaciones. Lo anterior refleja el papel de biblioteca escolar que las bibliotecas públicas han venido cumpliendo desde hace muchos años, lo cual también es resultado de la falta de estas en la Ciudad de México. En realidad esta situación no es mala si consideramos que la biblioteca pública está supliendo el papel de una institución importante como es la biblioteca escolar; sin embargo, de alguna manera se ha limitado a la atención y satisfacción de los usuarios de este tipo, descuidando a los demás integrantes de la sociedad, lo cual es preocupante dados los objetivos que tiene esta institución.

Un problema derivado del anterior es la falta de acervo actualizado, ya que todos estos usuarios asisten a las bibliotecas públicas en busca de información para sus tareas escolares, información de la que carecen la mayor parte de estos centros, ya que las remesas que manda la DGB son muy esporádicas y no contienen los materiales requeridos.

Otro aspecto que afecta a una gran parte de las bibliotecas públicas es el personal que trabaja en ellas, pues una gran mayoría no es del área bibliotecológica o de ciencias de la información, y además no está capacitado para trabajar en este tipo de instituciones. Aunque en múltiples ocasiones se ha puesto de relieve que la actualización y profesionalización del personal bibliotecario es primordial para el mejoramiento de los servicios que se ofrecen a la población, y hasta el mismo Jorge von Ziegler, titular de la DGB de la SEP, ha reconocido su importancia y ha señalado su intención de poner en marcha la capacitación de los recursos humanos por medio de la planeación, actualización y ampliación de los cursos que se imparten a los bibliotecarios de todo el país. La capacitación que la DGB ofrece a este personal se refiere en mayor medida a aspectos técnicos del manejo de una

Scire. $13: 1$ (en.-jun. 2007) 51-66. ISSN 1135-3716. 
biblioteca y en menor medida a temáticas como el desarrollo de talleres y cursos para los usuarios. Todo esto, unido a la falta de motivación e interés del personal, nos da como resultado una baja calidad en los servicios.

Otro de los problemas a los que se enfrenta una gran parte de las bibliotecas es la falta de instalaciones adecuadas para proporcionar un buen servicio, debido a que muchas bibliotecas se ubicaron en instalaciones construidas con diferentes propósitos y al ser utilizadas como bibliotecas no fueron adecuadamente acondicionadas, lo cual implica en muchas ocasiones iluminación inadecuada para leer, lugares insuficientes para albergar colecciones y usuarios, espacios húmedos y fríos, y carencia de instalaciones básicas como los sanitarios.

Todos los aspectos antes mencionados son en términos generales los principales problemas que tienen las bibliotecas públicas de la Ciudad de México; otros inconvenientes menos frecuentes son falta de fotocopiadoras, acervo en malas condiciones, inexistencia de conservación y restauración de libros, escasez de mobiliario, etcétera.

La figura 1 nos muestra el porcentaje de las bibliotecas en las que se conoce la información referente a la construcción de la megabiblioteca José Vasconcelos.

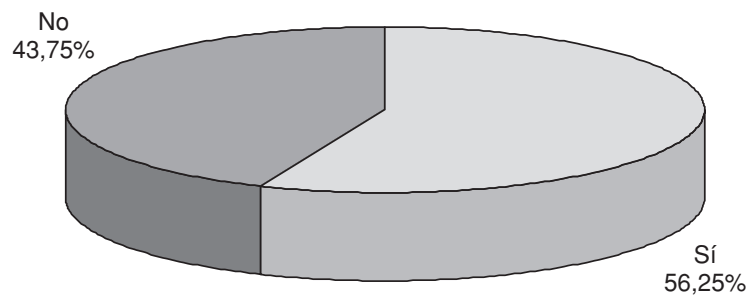

Figura 1. Porcentaje de bibliotecarios que conocen el proyecto de la megabiblioteca José Vasconcelos.

Como puede observarse, más de la mitad de los encargados han tenido alguna información acerca de este proyecto, lo que nos da la impresión inicial de que este ha sido ampliamente difundido por las autoridades del Conaculta y de que se han dado a conocer todos sus pormenores, como objetivos, servicios que ofrecerá, ubicación, etcétera, así como la manera en que la construcción de este nuevo edificio afectará al funcionamiento de toda la Red Nacional de Bibliotecas Públicas y a través de qué medios cumplirá los objetivos establecidos.

Las cifras de la figura 2 nos muestran que en realidad la información que tienen la mayor parte de los encargados es bastante superficial, ya que la categoría con el mayor porcentaje se refiere a que la megabiblioteca tendrá una colección muy grande y completa, sin especificar qué tipo de material tendrá, ni el acervo inicial 


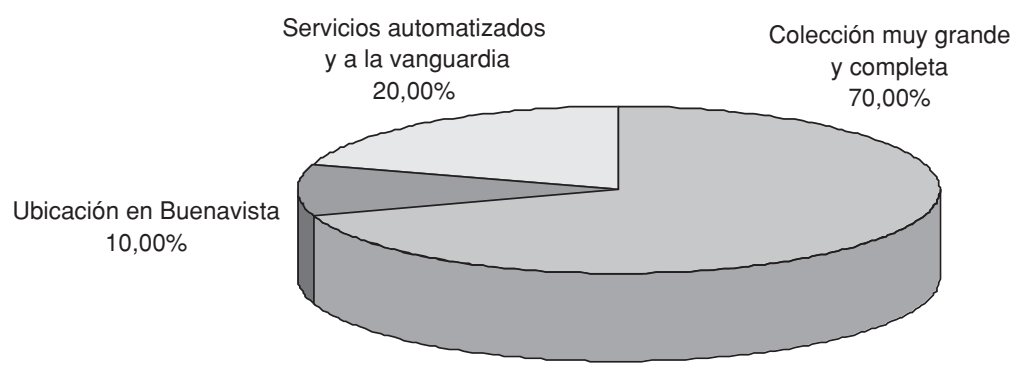

Figura 2. Información que se conoce del proyecto.

con que contará, ni la cantidad de materiales que podrá albergar aproximadamente. En las siguientes categorías solo encontramos información mínima acerca de los servicios automatizados y la ubicación física del inmueble. Todo ello nos indica que en realidad el Conaculta no ha informado de manera oficial a todos los integrantes de la Red Nacional, lo que parece desconcertante, pues, tratándose de un proyecto que implicará la participación de todos los ámbitos de la Red, es inconcebible que no se informe ampliamente de los alcances y objetivos de este.

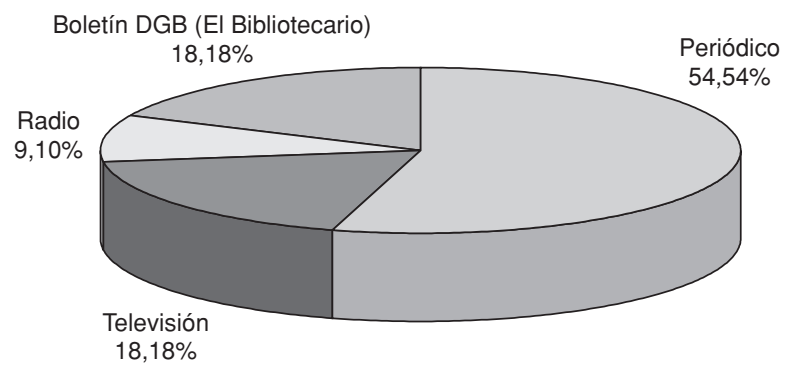

Figura 3. Medios por los que se enteraron de este proyecto.

Esta apreciación es apoyada por los datos presentados en la figura 3, que nos muestra que el medio por el que la mayor parte de los bibliotecarios obtuvo la información mencionada fue el periódico, y en menor medida la televisión, el boletín de la DGB El Bibliotecario y, finalmente, la radio, lo que indica claramente que el personal que trabaja en estas bibliotecas se ha enterado por medios externos.

Respecto a la pregunta de si estaban o no de acuerdo con la construcción de la megabiblioteca (figura 4), la mayor parte de los bibliotecarios manifestó su desacuerdo, pues su respuesta se basó en las carencias que tienen la mayoría de las bibliotecas públicas. Esta situación fue planteada en el diagnóstico presentado en 


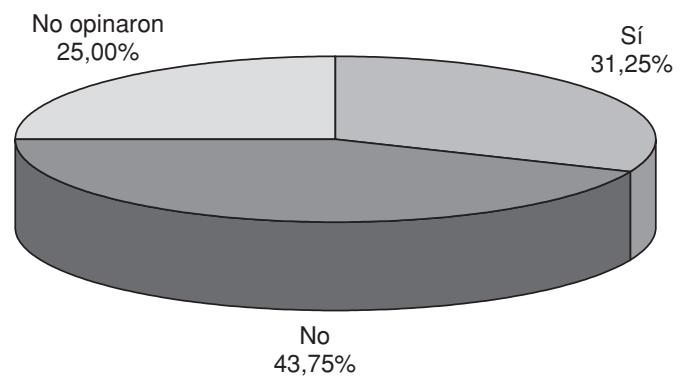

Figura 4. Bibliotecarios que estaban de acuerdo con este proyecto.

el Plan Nacional de Desarrollo 2001-2006, que fue corroborado ampliamente durante las visitas realizadas a diferentes bibliotecas. Las personas que dijeron estar de acuerdo con este proyecto se apoyaron sobre todo en las expectativas de contar con una biblioteca "modelo" que ayudará al mejoramiento de las otras bibliotecas y a la cual podrán remitir a todos los usuarios que lo requieran. Un porcentaje pequeño de bibliotecarios prefirieron no opinar al respecto, pues mencionaron que no tenían la información suficiente para formarse una opinión.

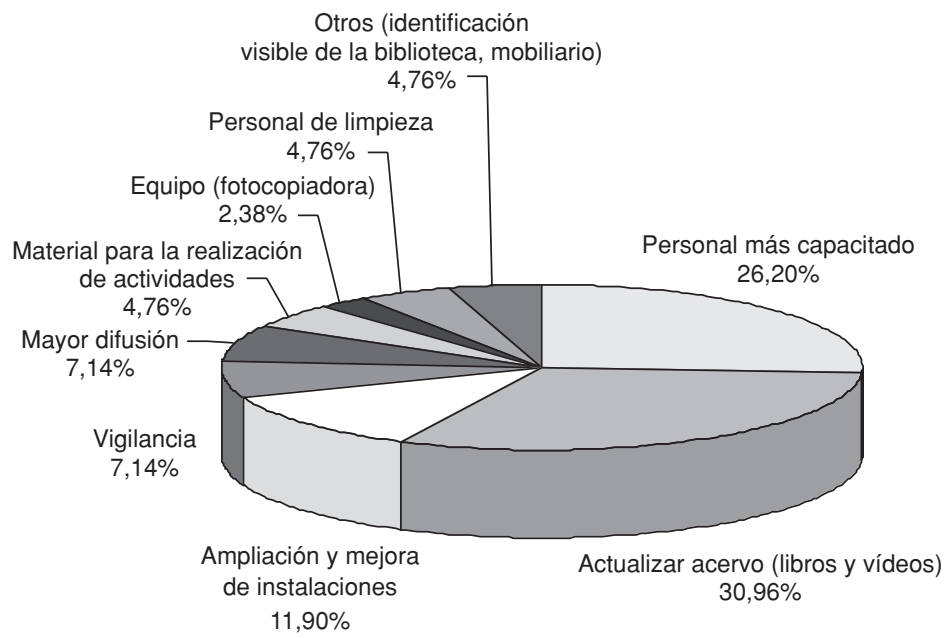

Figura 5. Necesidades básicas de la Red de Bibliotecas Públicas de la Ciudad de México.

Resultaron un poco desconcertantes las respuestas que apoyaron la construcción de la nueva megabiblioteca, sobre todo al conocerse las carencias en las que 
los encargados hubieran podido invertir un presupuesto como el que se asignó al proyecto de la construcción del nuevo edificio, tales como actualización de acervos (libros y vídeos) o capacitación del personal, así como también ampliación y mejoramiento de instalaciones, contratación de personal de vigilancia y empleados que realicen la limpieza, difusión de las bibliotecas, material para realizar actividades infantiles, servicio de fotocopias, y mobiliario nuevo y adecuado. Como se puede observar, los aspectos mencionados se relacionan con todas las deficiencias y problemas que presentan las bibliotecas públicas.

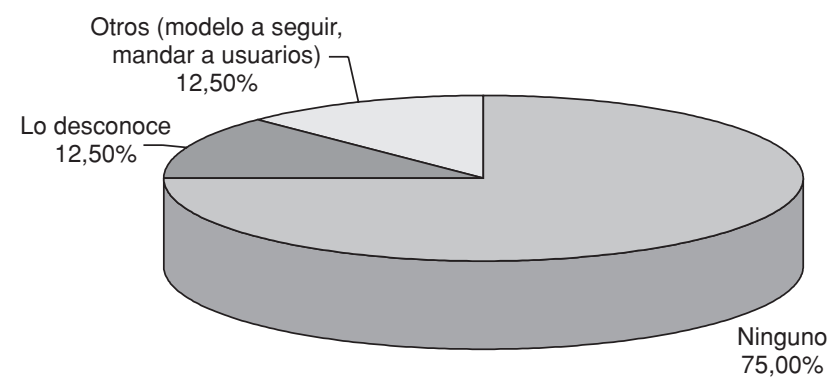

Figura 6. Beneficios que se obtendrán con la megabiblioteca José Vasconcelos.

Finalmente, desde el punto de vista de los encargados de estas bibliotecas, el nuevo proyecto de la megabiblioteca no aportará ningún beneficio a la Red Nacional de Bibliotecas Públicas, ya que no le ayudará a resolver los problemas que a diario tiene a causa de las carencias ya mencionadas.

Resumiendo la situación que viven las bibliotecas podemos enumerar los principales problemas a los que se enfrentan: $a$ ) las bibliotecas públicas han absorbido en gran medida las funciones de las bibliotecas escolares, las cuales son muy escasas; $b$ ) el personal que trabaja en las bibliotecas públicas tiene niveles insuficientes de capacitación y escasas oportunidades de superación y profesionalización; c) muchas de las bibliotecas necesitan mejorar sus instalaciones, ya que no tienen iluminación suficiente, no cuentan con sanitarios y en general carecen de los elementos necesarios para crear un espacio agradable.

Teniendo en cuenta este panorama, los encargados de las bibliotecas públicas opinan que no les aportaría ningún beneficio la creación de la megabiblioteca, y que en cambio con ese presupuesto se podrían mejorar muchos aspectos de las bibliotecas de la Ciudad de México.

\section{Conclusiones}

Conforme al objetivo de esta investigación, podemos concluir que en las bibliotecas públicas de la Ciudad de México existen necesidades básicas importantes Scire. $13: 1$ (en.-jun. 2007) 51-66. ISSN 1135-3716. 
que aún no son atendidas por falta de recursos económicos, por lo que resulta ilógico invertir en la construcción de un nuevo edificio para la nueva megabiblioteca José Vasconcelos. Falta mucho para que todas las bibliotecas puedan contar con un buen acervo que responda a las necesidades de información de la comunidad, así como tener suficiente presupuesto para la contratación de personal profesional en el área de bibliotecología, como también para la capacitación del personal ya existente. Todas estas carencias "han pasado desapercibidas" para el Conaculta, el cual ha impulsado la creación de esta biblioteca como un aspecto de primordial importancia para cumplir con el Programa Nacional de Cultura 2001-2006, olvidando que una biblioteca puede satisfacer más a su comunidad haciendo poco y bien que haciendo mucho y con deficiencias.

\section{Recomendaciones}

El Conaculta debería establecer prioridades con base en las necesidades reales de la Red Nacional de Bibliotecas Públicas.

Asimismo, habría que tener en cuenta el diagnóstico y los planes de trabajo correspondientes a la Red Nacional de Bibliotecas Públicas ya presentados en el Programa Nacional de Cultura 2001-2006, los cuales forman parte del Plan Nacional de Desarrollo, pues estos se realizan precisamente con el fin de identificar las debilidades de un proyecto o institución y establecer líneas de acción para subsanarlas.

\section{Referencias}

Amador, Judith; Ponce, Roberto (2003). Megabiblioteca: el descontento. // Proceso. (7 de julio de 2003) 60-61.

Anuncian partida de \$250 millones para etapa inicial de la construcción de la megabiblioteca (2003). // Jornada. 1388 (20 de marzo de 2003) 3A.

Busha, Charles (1990). Métodos de investigación en bibliotecología: técnicas e interpretación. México: UNAM, 1990.

Consejo Nacional para la Cultura y las Artes (México). Dirección General de Bibliotecas (199-). Directorio. México: Conaculta, 199-.

Consejo Nacional para la Cultura y las Artes (México) (2001). Programa Nacional de Cultura 2001-2006: La cultura en tus manos. México: Conaculta, 2001.

Editorial: la nueva sede de la Biblioteca de México (2003). // El Bibliotecario. 3:30 (2003) 16.

La editorial alemana Taschen donó una importante colección de libros para la nueva Biblioteca de México José Vasconcelos (2004). // El Bibliotecario. 3:35 (2004) 7.

Encuentran confusión en plan modernizador (2002). // Reforma. (3 de diciembre de 2002) 3C.

Hay déficit de bibliotecarios en México: Nahum Pérez (2001). // El Universal. (13 de junio de 2001) F1-F2.

Palacios, Cynthia (2001). Analizarán retos de bibliotecas. // El Universal. (7 de junio de 2001) F1.

Scire. $13: 1$ (en.-jun. 2007) 51-66. ISSN 1135-3716. 
Piden nuevo edificio para "megabiblioteca" (2002). // Reforma. (3 de diciembre de 2002) 3C.

Poniatowska, Elena (2003). Buenavista, en vías de volverse un monumento a la vanidad. // Jornada. 1391 (10 de junio de 10, 2003) 2A, 4A.

Poniatowska, Elena (2003). La lectura en voz alta es mejor que un edificio apantallador. // Jornada. 1391 (11 de junio de 2003) 3A.

El Programa Nacional de Bibliotecas Públicas 1983-1988 y el Centro Bibliotecario Nacional Biblioteca Pública de México (1988). México: SEP, 1988.

La Red Nacional de Bibliotecas Públicas (1991). México: Conaculta, 1991.

Requiere especialistas "megabiblioteca" (2002). // Reforma. (3 de diciembre de 2002) 3C.

Se instaló el Comité Consultivo del proyecto del nuevo edificio de la Biblioteca de México José Vasconcelos (2003). // El Bibliotecario. 2:22 (2003) 1-3.

Se presentó el proyecto de la nueva Biblioteca José Vasconcelos a los miembros de la Asociación Mexicana de Bibliotecarios (2003). // El Bibliotecario. 3:30 (2003) 1-6.

Sigue "megabiblioteca" modelos nórdicos (2003). // Reforma. (7 de julio de 2003) 3C. Tachan de equívoco crear megabiblioteca (2002). // Reforma. (17 de julio de 2002) 2C.

\section{Anexo}

Cuestionario: entrevista para los encargados de las bibliotecas públicas de las redes delegacionales de la Ciudad de México

1. ¿Conoce usted el proyecto de la construcción de la megabiblioteca José Vasconcelos?

Sí

No

2. ¿Qué información concreta conoce de este proyecto?

3. ¿Cuál es el medio por el que llegó a usted esta información?

4. ¿Está de acuerdo con la construcción de esta biblioteca? Sí _ No ¿Por qué?

5. ¿De tener la autoridad para hacerlo, en qué utilizaría el dinero destinado a este proyecto dentro de las bibliotecas ya existentes? 
6. ¿Qué beneficios cree usted que obtendrá la Red Nacional de Bibliotecas Públicas con la creación de esta biblioteca? 